\title{
Phase transitions in hybrid layered halide perovskites - Hydrogen bonding meets octahedral tilting
}

\author{
P. M. Woodward, N. P. Holzapfel, T. Liu, J. Race, A. Milder \\ ${ }^{1}$ Department of Chemistry and Biochemistry, The Ohio State University, $100 \mathrm{~W} .18^{\text {th }}$ Avenue, Columbus, OH 43210, USA \\ Woodward.55@osu.edu
}

Using the tools of group theory, we have mapped out the symmetry consequences of structural distortions in hybrid layered perovskites. These distortions include octahedral tilting and cation ordering within the inorganic layers as well as orientational ordering of organic cations in the organic layers. This analysis is coupled with synthesis and crystal structure determination of a wide variety Ruddlesden-Popper phases. The combined analysis shows that certain modes of octahedral tilting are strongly favored in large part because they lead to favorable hydrogen bonding interactions between the halide ions of the inorganic layers and the ammoniumtype protons of the organic cations. This information is used to guide our search for new lead-free ferroelectrics and multiferroics among this large class of compounds.

Keywords: perovskites, phase transitions, halides, layered materials 